\title{
THERMAL SHOCK IN AN ELASTIC BODY WITH A SPHERICAL CAVITY*
}

\author{
BY \\ ELI STERNBERG AND J. G. CHAKRAVORTY \\ Brown University
}

Summary. This investigation aims at the dynamic thermoelastic response of an infinite medium with a spherical cavity to a sudden uniform change in the temperature of its internal boundary. By means of the Laplace transform, a closed solution to this problem-exact within classical elastokinetics-is obtained in terms of error functions of real and complex arguments. The ensuing temperature stresses are compared with the corresponding quasi-static results.

Introduction. The conventional quasi-static treatment of transient thermoelastic problems rests on the assumption, apparently introduced first by Duhamel [1] ${ }^{1}$, that the inertia terms may be neglected in the governing field equations. The quality of the approximate solutions thus obtained evidently depends both upon the magnitude of the time-gradients inherent in the temperature field and upon the size of the relevant intrinsic inertia parameters.

Duhamel's hypothesis has been the object of several recent studies. The earliest investigation of this kind is due to Danilovskaya [2] (1950), who determined the dynamic thermal stresses induced in an elastic half-space by a sudden uniform heating (or cooling) of the entire boundary, if the body is constrained to a uniaxial motion perpendicular to the bounding plane ${ }^{2}$. Later on, Danilovskaya [4] generalized her solution to accommodate convective boundary conditions. Still more recently, the present authors [5] established explicitly the thermal displacements associated with the stresses given in [2], [3] and extended the completed solution to the case of a gradual (ramp-type) change of the surface-temperature. Related studies in the theory of beams and plates were carried out by Boley [6] and by Boley and Barber [7]. Finally, we mention thermoelastic investigations by Nowacki [8] and Ignaczak [9] in which the inertia terms are taken into account, although neither of these papers is concerned with a quantitative assessment of the dynamic effects thus arising.

As was pointed out in [5], the problem treated in [2], [3], [5] suffers from two deficiencies as a vehicle for examining the adequacy of the traditional quasi-static methods in transient thermoelasticity. First, the artificial constraint of the medium imposed in [2] results in a severe degeneracy of the corresponding quasi-static stress distribution, the normal stress perpendicular to the boundary being identically zero. Second, the absence of a characteristic length in this particular problem causes the pertinent inertia parameter to be hidden in the dimensionless time and distance scales whence its influence upon the dynamic effects sought, is obscured.

For the preceding reasons it appears desirable to examine the inertia effects en-

\footnotetext{
*Received July 1, 1958. The results communicated in this paper were obtained in the course of an investigation conducted under Contract Nonr 562(25) with the Office of Naval Research, Department of the Navy, Washington, D. C.

${ }^{1}$ Numbers in brackets refer to the list of references at the end of the paper.

${ }^{2}$ The results contained in [2] were independently reached by Mura [3] (1952).
} 
countered in a less degenerate thermoelastic space problem. This leads us to consider an infinite, homogeneous and isotropic, elastic medium with a spherical cavity, the boundary of which is exposed to an instantaneous change of temperature. We assume that the body, which is free from loading, is initially at rest, and seek the ensuing thermal stresses and displacements if the temperature field obeys the (uncoupled) heat-conduction equation. The corresponding quasi-static solution was given and discussed in [10]. It should be mentioned that the present dynamic thermoelastic problem is related to an ordinary problem of elastokinetics, i.e. to the determination of the stresses produced by a sudden uniform pressure applied to the surface of a spherical cavity in an infinite elastic medium. An exact closed solution to this more elementary problem was obtained first by Jeffreys [11] (1931) and has since been rediscovered by various subsequent authors.

Formulation of problem. Dimensionless variables. Let $(r, \theta, \varphi)$ respectively denote the radial coordinate, the co-latitude, and the longitude of a spherical coordinate system. Suppose that the medium under consideration occupies the region $a \leq r<\infty$, where $a$ is the radius of the spherical cavity. The thermoelastic problem described in the Introduction is characterized by polar symmetry about the origin, whence

$$
T=T(r, t) ; \quad u_{r}=u(r, t), \quad u_{\theta}=u_{\varphi}=0,
$$

in which $t$ is the time, $T$ the temperature, while $\left(u_{r}, u_{\theta}, u_{\varphi}\right)$ designate the spherical components of the displacement vector.

If $T_{0}$ is the constant temperature suddenly assumed by the internal boundary $r=a$, and $\kappa$ stands for the thermal diffusivity of the material, we may introduce a dimensionless radial coordinate, time and temperature, by means of

$$
\rho=\frac{r}{a}, \quad \tau=\frac{\kappa t}{a^{2}}, \quad \varphi=\frac{T}{T_{0}} .
$$

With reference to these dimensionless variables, the independent temperature problem is governed by the (uncoupled) heat-conduction equation

$$
\frac{\partial^{2} \varphi}{\partial \rho^{2}}+\frac{2}{\rho} \frac{\partial \varphi}{\partial \rho}=\frac{\partial \varphi}{\partial \tau}
$$

subject to the initial condition

$$
\varphi(\rho, 0)=0,
$$

together with the boundary and regularity conditions

$$
\varphi(1, \tau)=h(\tau), \quad \varphi(\rho, \tau) \rightarrow 0 \quad \text { as } \rho \rightarrow \infty,
$$

where $h(\tau)$ is the Heaviside step-function, given by

$$
h(\tau)=0 \text { for }-\infty<\tau<0, \quad h(\tau)=1 \text { for } 0<\tau<\infty .
$$

We turn next to the characterization of the thermal displacements and stresses associated with the temperature field $\varphi(\rho, \tau)$. To this end, let a dimensionless radial displacement $u^{*}$, as well as dimensionless spherical components of normal stress $\sigma_{r}^{*}$ and $\sigma_{\theta}^{*}$, be defined through 


$$
\left.\begin{array}{c}
u^{*}=\frac{1-\nu}{(1+\nu) \alpha T_{0} a} u, \\
\sigma_{r}^{*}=\frac{1-\nu}{2(1+\nu) \alpha T_{0} \mu} \sigma_{r}, \quad \sigma_{\theta}^{*}=\frac{1-\nu}{2(1+\nu) \alpha T_{0} \mu} \sigma_{\theta}
\end{array}\right\}
$$

in which $\sigma_{r}, \sigma_{\theta}$ are the corresponding physical components of normal stress, whereas $\mu, \nu$, and $\alpha$ denote the shear modulus, Poisson's ratio, and the coefficient of thermal expansion, respectively. In view of (1), the spherical components of shear stress vanish identically and $\sigma_{\varphi}=\sigma_{\theta}$. Finally, it is expedient to introduce a dimensionless inertia parameter $\gamma$, defined by

$$
\gamma=\frac{\kappa}{c a}
$$

where $c$ is the velocity of irrotational waves,

$$
c^{2}=\frac{2(1-\nu) \mu}{(1-2 \nu) \beta},
$$

$\beta$ being the mass density of the material.

The displacement equation of motion now takes the form

$$
\frac{\partial^{2} u^{*}}{\partial \rho^{2}}+\frac{2}{\rho} \frac{\partial u^{*}}{\partial \rho}-\frac{2 u^{*}}{\rho^{2}}=\gamma^{2} \frac{\partial^{2} u^{*}}{\partial \tau^{2}}+\frac{\partial \varphi}{\partial \rho},
$$

while the stress-displacement relations become

$$
\left.\begin{array}{l}
(1-2 \nu) \sigma_{r}^{*}=(1-\nu) \frac{\partial u^{*}}{\partial \rho}+2 \nu \frac{u^{*}}{\rho}-(1-\nu) \varphi, \\
(1-2 \nu) \sigma_{\theta}^{*}=\nu \frac{\partial u^{*}}{\partial \rho}+\frac{u^{*}}{\rho}-(1-\nu) \varphi .
\end{array}\right\}
$$

Once $\varphi(\rho, \tau)$ is known, the solution of the thermoelastic problem at hand reduces to the determination of a function $u^{*}(\rho, \tau)$ which satisfies (10), meets the initial conditions

$$
u^{*}(\rho, 0)=0, \quad\left[\frac{\partial u^{*}}{\partial \tau}\right]_{(\rho, 0)}=0,
$$

and is such that the stresses (11) conform to the boundary condition

$$
\sigma_{r}^{*}(1, \tau)=0
$$

as well as to the regularity conditions

$$
\sigma_{r}^{*}(\rho, \tau) \rightarrow 0, \quad \sigma_{\theta}^{*}(\rho, \tau) \rightarrow 0 \quad \text { as } \rho \rightarrow \infty .
$$

We observe that the quasi-static formulation of the present problem ${ }^{3}$ is obtained by setting $\gamma$ equal to zero in (10) and by deleting (12).

Introduction of the Laplace transform. Solution in the transform domain. The solution of the elementary heat-conduction problem governed by (3), (4), (5), is well known and admits the closed representation 


$$
\varphi(\rho, \tau)=\frac{1}{\rho} \psi(\xi), \quad \xi=\frac{\rho-1}{2(\tau)^{1 / 2}}
$$

where

$$
\psi(\xi) \equiv \operatorname{erfc}(\xi)=\frac{2}{\pi^{1 / 2}} \int_{\xi}^{\infty} \exp \left(-\eta^{2}\right) d \eta
$$

is the complementary error function. For future convenience we recall ${ }^{5}$ here that

$$
\psi(0)=1, \quad \psi(\xi) \rightarrow 0 \text { as } \xi \rightarrow \infty, \quad \psi(-\xi)=2-\psi(\xi),
$$

and cite the power-series development

$$
\psi(\xi)=1-\frac{2}{\pi^{1 / 2}} \sum_{n=0}^{\infty} \frac{(-1)^{n} \xi^{2 n+1}}{(2 n+1) \underline{n}},
$$

as well as the semi-convergent expansion, valid as $\operatorname{Re}(\xi) \rightarrow+\infty$,

$$
\psi(\xi)=\frac{\exp \left(-\xi^{2}\right)}{\pi^{1 / 2} \xi}\left[\sum_{n=0}^{N} \frac{\left.(-1)^{n}\right|^{2 n}}{\underline{\mid n}(2 \xi)^{2 n}}+O\left(\xi^{-2 N-3}\right)\right] \quad(N=1,2, \cdots) .
$$

With a view toward the determination of the thermal displacements and stresses arising from the temperature distribution (15), let

$$
U(\rho, s)=L\left\{u^{*}(\rho, \tau)\right\}=\int_{0}^{\infty} \exp (-s \tau) u^{*}(\rho, \tau) d \tau,
$$

so that $U(\rho, s)$ is the Laplace transform with respect to $\tau$ of $u^{*}(\rho, \tau), s$ being the transform parameter. From (15),

$$
\Phi(\rho, s)=L\{\varphi(\rho, \tau)\}=\frac{1}{\rho s} \exp \left[-(\rho-1) s^{1 / 2}\right],
$$

whence applying the transform to (10), and bearing in mind (12), we reach the ordinary differential equation

$$
\frac{d^{2} U}{d \rho^{2}}+\frac{2}{\rho} \frac{d U}{d \rho}-\left(\frac{2}{\rho^{2}}+\gamma^{2} s^{2}\right) U=-\left(\frac{1}{\rho s^{1 / 2}}+\frac{1}{\rho^{2} s}\right) \exp \left[-(\rho-1) s^{1 / 2}\right] .
$$

Furthermore, by virtue of (11) to (14), (20), (21), we arrive at the transformed boundary and regularity conditions

$$
\left.\begin{array}{c}
{\left[(1-\nu) \frac{d U}{d \rho}+2 \nu \frac{U}{\rho}-(1-\nu) \Phi\right]_{(1, s)}=0,} \\
U(\rho, s) \rightarrow 0 \text { as } \rho \rightarrow \infty .
\end{array}\right\}
$$

Equation (22) is reducible to an inhomogeneous modified Bessel equation. Its general solution is given by

$$
U=V(\rho, s)+W(\rho, s),
$$


where $W(\rho, s)$ is a particular integral of the complete equation, and

$$
V(\rho, s)=\frac{\lambda_{1}(s)}{\rho^{1 / 2}} I_{3 / 2}(\gamma s \rho)+\frac{\lambda_{2}(s)}{\rho^{1 / 2}} K_{3 / 2}(\gamma s \rho) .
$$

Here, $\lambda_{1}(s), \lambda_{2}(s)$ are arbitrary functions of $s$, while $I_{3 / 2}$ and $K_{3 / 2}$ designate modified Bessel functions of the first and second kind, respectively. As is readily confirmed, $W$ may be taken in the form

$$
W(\rho, s)=\frac{1+\rho s^{1 / 2}}{\rho^{2} s^{2}\left(\gamma^{2} s-1\right)} \exp \left[-(\rho-1) s^{1 / 2}\right] .
$$

On the other hand, (23) to (26), after an elementary computation, yield

$$
V(\rho, s)=\frac{(1+\gamma \rho s)\left\{\gamma^{2} s^{2}+2 p\left[1+s^{1 / 2}\right]\right\}}{\rho^{2} s^{2}\left(1-\gamma^{2} s\right)\left[(\gamma s+p)^{2}+q^{2}\right]} \exp [-\gamma(\rho-1) s],
$$

provided

$$
p=\frac{1-2 \nu}{1-\nu}, \quad q=\frac{(1-2 \nu)^{1 / 2}}{1-\nu} .
$$

Inverse transforms. Solution of problem. We now proceed to establish the desired inverse transform of $U(\rho, s)$. For this purpose we note from (26) that

$$
W=W_{1}(\rho, s) W_{2}(\rho, s),
$$

if

$$
W_{1}=\rho^{-2}\left(\gamma^{2} s-1\right)^{-1}, \quad W_{2}=\left(s^{-2}+\rho s^{-3 / 2}\right) \exp \left[-(\rho-1) s^{1 / 2}\right] .
$$

Moreover, according to known results?

$$
\left.\begin{array}{l}
w_{1}^{*}(\rho, \tau)=L^{-1}\left\{W_{1}(\rho, s)\right\}=\frac{1}{\gamma^{2} \rho^{2}} \exp \left(\tau / \gamma^{2}\right), \\
w_{2}^{*}(\rho, \tau)=L^{-1}\left\{W_{2}(\rho, s)\right\}=(\rho+1)(\tau / \pi)^{1 / 2} \exp \left(-\xi^{2}\right)+\frac{1-\rho^{2}}{2} \psi(\xi)+\tau \psi(\xi),
\end{array}\right\}
$$

where $\xi$ and $\psi(\xi)$ are defined in (15), (16). From (29), (31), and the convolution theorem, follows

$$
w^{*}(\rho, \tau)=L^{-1}\{W(\rho, s)\}=\int_{0}^{\tau} w_{1}^{*}(\rho, \tau-\eta) w_{2}^{*}(\rho, \eta) d \eta .
$$

Substitution of (31) into (32), and repeated use of integration by parts, ultimately lead to

$$
\left.\begin{array}{rl}
w^{*}(\rho, \tau)=\frac{1}{2 \rho^{2}}\left[\left(\rho^{2}-\right.\right. & \left.2 \tau-1-2 \gamma^{2}\right) \psi(\xi) \\
& \left.-2(\rho+1)(\tau / \pi)^{1 / 2} \exp \left(-\xi^{2}\right)+\gamma^{2} \psi_{1}(\rho, \tau)-\gamma \rho \psi_{2}(\rho, \tau)\right] .
\end{array}\right\}
$$

The auxiliary functions $\psi_{1}, \psi_{2}$ appearing in (33) are accounted for by

7See [14], No. 1, p. 229; No. 4, p. 245; No. 7, p. 246. 


$$
\begin{aligned}
& \psi_{1}(\rho, \tau)=\psi\left(\xi+\frac{\tau^{1 / 2}}{\gamma}\right) \exp \left(\frac{\tau}{\gamma^{2}}+\frac{\rho-1}{\gamma}\right) \\
& +\psi\left(\xi-\frac{\tau^{1 / 2}}{\gamma}\right) \exp \left(\frac{\tau}{\gamma^{2}}-\frac{\rho-1}{\gamma}\right)=\gamma \frac{\partial \psi_{2}}{\partial \rho}, \\
& \psi_{2}(\rho, \tau)=\psi\left(\xi+\frac{\tau^{1 / 2}}{\gamma}\right) \exp \left(\frac{\tau}{\gamma^{2}}+\frac{\rho-1}{\gamma}\right) \\
& -\psi\left(\xi-\frac{\tau^{1 / 2}}{\gamma}\right) \exp \left(\frac{\tau}{\gamma^{2}}-\frac{\rho-1}{\gamma}\right) .
\end{aligned}
$$

Our next task consists in finding the inverse Laplace transform of $V(\rho, s)$ in (27). Evidently,

with

$$
v^{*}(\rho, \tau)=L^{-1}\{V(\rho, s)\}=h(\omega) L^{-1}\{G(\rho, s)\}
$$

$$
\omega=\tau-\gamma(\rho-1),
$$

if $h$ again denotes the Heaviside step-function given by (6), and $G$ is the coefficient of the exponential function in (27). Moreover, a partial fraction decomposition of $G(\rho, s)$ results in

$$
G(\rho, s)=\frac{\gamma^{2}+2 p s^{-2}+2 p s^{-3 / 2}}{\gamma q \rho^{2}\left(1+2 \gamma p+2 \gamma^{2} p\right)} \operatorname{Re}\left[\frac{F(\rho)}{s-\gamma^{-2}}-\frac{F(\rho)}{s-k}\right],
$$

where "Re" stands for "the real part of", $k$ is the complex parameter defined by

$$
k=\frac{-p+i q}{\gamma},
$$

and

$$
F(\rho)=-q(\gamma+\rho)+i[1+p(\gamma-2 \gamma \rho-\rho)] .
$$

The inverse of $G(\rho, s)$ is readily found with the aid of the available inverse transform

$$
L^{-1}\left\{\frac{s^{-3 / 2}}{s-\lambda}\right\}=\lambda^{-3 / 2}\left[1-\psi\left(\lambda^{1 / 2} \tau^{1 / 2}\right)\right] \exp (\lambda \tau)-\frac{2}{\lambda}\left(\frac{\tau}{\pi}\right)^{1 / 2},
$$

in which $\lambda$ is a constant. Thus, (35) and (37) imply

$$
\left.\begin{array}{rl}
v^{*}(\rho, \tau)=-\frac{h(\omega)}{\rho^{2}} & {\left[\gamma(\gamma+\rho) \exp \left(\omega / \gamma^{2}\right)\right.} \\
+ & A \operatorname{Re}\left\{F ( \rho ) \left[\gamma^{3} \psi\left(\omega^{1 / 2} / \gamma\right) \exp \left(\omega / \gamma^{2}\right)-k^{-3 / 2} \psi(k \omega)^{1 / 2} \exp (k \omega)\right.\right. \\
+ & \left.\left.\left.B \exp (k \omega)+C\left[\omega+2(\omega / \pi)^{1 / 2}\right]+D\right]\right\}\right] .
\end{array}\right\}
$$

The letters $A, B, C, D$, appearing in (41), refer to the following auxiliary functions of $\nu$ and $\gamma$ :

${ }^{8}$ Note that the arguments of the complementary error function $\psi$ here are $\xi+\tau^{\frac{1}{2}} / \gamma$ or $\xi-\tau^{\frac{1}{2}} / \gamma$, as indicated.

${ }^{9}$ See [14], No. 2, p. 233.

${ }^{10}$ Here, as well as subsequently, $k^{\frac{1}{3}}$ stands for the principal value of this root. 


$$
\left.\begin{array}{c}
A=\frac{2 p}{\gamma q\left(1+2 \gamma p+2 \gamma^{2} p\right)}, \\
B=\frac{\gamma^{2}}{2 p}+k^{-2}+k^{-3 / 2}, \quad C=\gamma^{2}-k^{-1}, \quad D=\gamma^{4}-k^{-2} .
\end{array}\right\}
$$

From (24), (32), (35), we have

$$
u^{*}=v^{*}(\rho, \tau)+w^{*}(\rho, \tau),
$$

where $v^{*}$ and $w^{*}$ are given by (41) and (33).

The thermal stresses associated with the displacement (43), are obtained by substitution of (43) into (11). If

$$
E=\frac{\partial F}{\partial \rho}=-q-i(1+2 \gamma) p,
$$

the results of these lengthy computations become:

$$
\begin{aligned}
\sigma_{r}^{*}=\frac{h(\omega)}{p \rho^{3}}\left[\left[\rho^{2}\right.\right. & +2 \gamma p(\gamma+\rho)] \exp \left(\omega / \gamma^{2}\right) \\
& +A \operatorname{Re}\left\{[F(\rho)(\rho+2 \gamma p)-E \gamma \rho] \gamma^{2} \psi\left(\omega^{1 / 2} / \gamma\right) \exp \left(\omega / \gamma^{2}\right)\right. \\
& +[E \rho-F(\rho)(2 p+\gamma k \rho)] k^{-3 / 2} \psi\left(k^{1 / 2} \omega^{1 / 2}\right) \exp (k \omega) \\
& +B[F(\rho)(2 p+\gamma k \rho)-E \rho] \exp (k \omega) \\
& +C[2 p F(\rho)-E \rho]\left[\omega+2(\omega / \pi)^{1 / 2}\right] \\
& +F(\rho)(2 p D+\gamma \rho C)-D E \rho\}] \\
& +\frac{1}{\rho^{3}}\left[\left(2 \tau+2 \gamma^{2}+1-\rho^{2}\right) \psi(\xi)+2(\rho+1)(\tau / \pi)^{1 / 2} \exp \left(-\xi^{2}\right)\right. \\
& \left.-\left(\frac{\rho^{2}}{2 p}+\gamma^{2}\right) \psi_{1}(\rho, \tau)+\gamma \rho \psi_{2}(\rho, \tau)\right],
\end{aligned}
$$

and, setting

$$
\begin{aligned}
& m=\frac{\nu}{1-2 \nu}=\frac{q^{2}}{p^{2}}-\frac{1}{p} \\
& \sigma_{\theta}^{*}=\frac{h(\omega)}{\rho^{3}}\left[\left[m \rho^{2}-\gamma(\gamma+\rho)\right] \exp \left(\omega / \gamma^{2}\right)\right. \\
& +A \operatorname{Re}\left\{[F(\rho)(m \rho-\gamma)-E \gamma m \rho] \gamma^{2} \psi\left(\omega^{1 / 2} / \gamma\right) \exp \left(\omega / \gamma^{2}\right)\right. \\
& +\left[E m \rho-F(\rho)(\gamma m k \rho-1) k^{-3 / 2} \psi\left(k^{1 / 2} \omega^{1 / 2}\right) \exp (k \omega)\right. \\
& +B[F(\rho)(\gamma m k \rho-1)-E m \rho] \exp (k \omega) \\
& -C[F(\rho)+E m \rho]\left[\omega+2(\omega / \pi)^{1 / 2}\right] \\
& +F(\rho)(C \gamma m \rho-D)-D E m \rho\}] \\
& -\frac{1}{2 \rho^{3}}\left[\left(2 \tau+2 \gamma^{2}+1+\rho^{2}\right) \psi(\xi)+2(\rho+1)(\tau / \pi)^{1 / 2} \exp \left(-\xi^{2}\right)\right. \\
& \left.-\left(\gamma^{2}-m \rho^{2}\right) \psi_{1}(\rho, \tau)+\gamma \rho \psi_{2}(\rho, \tau)\right] \text {. }
\end{aligned}
$$


This completes the formal solution to the thermoelastic problem at hand. One confirms by direct substitution, with the aid of (17), (18), (19), that the displacement (43) and the stresses (45), (47), indeed satisfy the field equations (10), (11) if $\omega \neq 0$, and conform to conditions (12), (13), (14).

Properties of solution. Quasi-static and steady-state limits. Examining the structure of the solution obtained in the preceding section, we observe that the displacement (43) and the stresses (45), (47), each contain a term which has the step-function $h(\omega)$ as a multiplier. If $\gamma>0$, these terms reflect the presence of a spherical shock-wave issuing from the boundary $\rho=1$ and propagating outward with the velocity $c$ of irrotational waves, given by (9). The remaining terms are diffusive in character and correspond to a signal which is instantaneously received throughout the entire medium.

In accordance with (6), (36), we have

$$
\tau=\gamma(\rho-1),
$$

provided $\rho$ and $\tau$ momentarily designate the dimensionless radius of the wave-front and the dimensionless time at which this radius is attained. While the time and space derivatives of the radial displacement suffer discontinuities at $\tau=\gamma(\rho-1), u^{*}$ itself depends continuously on $\rho$ and $\tau$ for $1<\rho<\infty, 0<\tau<\infty$, as required by the physical continuity of the material. On the other hand, the stresses exhibit finite jump-discontinuities at the wave-front. Specifically,

$$
\left.\begin{array}{l}
\sigma_{r}^{*}[\rho, \gamma(\rho-1)+]-\sigma_{r}^{*}[\rho, \gamma(\rho-1)-]=\frac{1-\nu}{(1-2 v) \rho}, \\
\sigma_{\theta}^{*}[\rho, \gamma(\rho-1)+]-\sigma_{\theta}^{*}[\rho, \gamma(\rho-1)-]=\frac{\nu}{(1-2 \nu) \rho} \cdot
\end{array}\right\}
$$

Thus, $\sigma_{\theta}^{*}$ remains continuous only if $\nu=0$; the jumps in both stresses are independent of the inertia parameter $\gamma$ and become unbounded as $\nu \rightarrow 1 / 2$, if $\mu$ remains fixed in this limit.

It is interesting to compare (49) with the stress-discontinuities generated in Jeffreys' problem [11] by a radial pressure of magnitude $\sigma_{0}$, which is suddenly applied to the spherical boundary and is steadily maintained thereafter. Taking, in this instance, $\tau=c t / a, \sigma_{r}^{*}=\sigma_{r} / \sigma_{0}, \sigma_{\theta}^{*}=\sigma_{\theta} / \sigma_{\sigma_{i}}$, one has here

$$
\left.\begin{array}{l}
\sigma_{r}^{*}[\rho,(\rho-1)+]-\sigma_{r}^{*}[\rho,(\rho-1)-]=-\frac{1}{\rho}, \\
\sigma_{\theta}^{*}[\rho,(\rho-1)+]-\sigma_{\theta}^{*}[\rho,(\rho-1)-]=-\frac{\nu}{(1-\nu) \rho} \cdot
\end{array}\right\}
$$

In contrast, the stress-discontinuities arising from the thermal shock in Danilovskaya's problem [2] of the half-space are independent of the position of the plane wave-front and persist undiminished for all time.

As the inertia parameter $\gamma$ approaches zero, while $\rho$ and $\tau$ are held fixed, the dynamic solution to the present problem should tend to the corresponding quasi-static solution. Carrying out this cumbersome limit process, which necessitates repeated use of the properties of $\psi$ cited in (17), (18), (19), we obtain: 


$$
\left.\begin{array}{c}
u^{*}=\frac{1}{\rho^{2}} \Psi(\rho, \tau), \\
\sigma_{r}^{*}=-\frac{2}{\rho^{3}} \Psi(\rho, \tau), \quad \sigma_{\theta}^{*}=\frac{1}{\rho^{3}} \Psi(\rho, \tau)-\varphi(\rho, \tau),
\end{array}\right\}
$$

where

$$
\left.\begin{array}{rl}
\Psi(\rho, \tau) & =\int_{1}^{\rho} \eta^{2} \varphi(\eta, \tau) d \eta \\
& =\frac{1}{2}\left(\rho^{2}-2 \tau-1\right) \psi(\xi)-(\rho+1)(\tau / \pi)^{1 / 2} \exp \left(-\xi^{2}\right)+\tau+2(\tau / \pi)^{1 / 2} .
\end{array}\right\}
$$

Equations (51), (52) are identical with the quasi-static results deduced directly in [10]. As pointed out in [10], the displacement $u^{*}$ in (51) vanishes identically on the boundary $\rho=1$, which remains fixed for all time. This curious conclusion fails to apply once inertia effects are taken into account. It should be mentioned that a quasi-static solution (in infinite series form) to the companion problem of a solid sphere whose surface temperature is suddenly altered, was given by Grünberg [15], as early as 1925; the same problem was reconsidered by Melan [16], [17]. Trostel [18] presented a formal quasi-static series solution to the general axisymmetric transient thermal-stress problem for a spherical shell of arbitrary thickness.

Next, consider the limit of (15), (43), (45), (47), as $\tau \rightarrow \infty$, for fixed $\rho$ and $\gamma$. Here we find, after a tedious computation,

$$
\left.\begin{array}{c}
\varphi=\frac{1}{\rho}, \quad u^{*}=\frac{1}{2}\left(1-\rho^{-2}\right), \\
\rho^{-3}-\rho^{-1}, \quad \sigma_{\theta}^{*}=-\frac{1}{2}\left(\rho^{-3}+\rho^{-1}\right) .
\end{array}\right\}
$$

These values of displacement and stresses also characterize the limit of (51) as $\tau \rightarrow 0$. Consequently, the dynamic and the quasi-static solution approach the same steady state. Formulas (53) are in agreement with the appropriate steady-state results for a spherical shell. ${ }^{11}$

Numerical results. Discussion. We turn, finally, to the discussion of certain numerical results based on the dynamic solution to the transient thermoelastic problem treated in this paper. Our main objective, in this connection, is to illustrate the character, and assess the magnitude, of the departures from the analogous quasi-static results. The space and time dependence of the temperature field (15), as well as of the quasi-static displacement and stresses (51), were discussed extensively in [10]; the corresponding diagrams need not be reproduced here.

The dynamic solution, in contrast to (51), depends not only on $\rho$ and $\tau$, but involves also the inertia parameter $\gamma$ and Poisson's ratio ${ }^{12} \nu$. An inspection of (43), (45), (47), together with the equations defining the various auxiliary functions, reveals that these formulas are expressible exclusively in terms of elementary functions and error functions of real argument, provided $\omega=\tau-\gamma(\rho-1)<0$. For $\tau>\gamma(\rho-1)$, however, that is, for points lying behind the wave-front, the displacement and stresses contain, in

${ }^{11}$ See, for example, [19], p. 420. See also [20].

${ }^{12}$ Note that the dimensionless quantities $u^{*}, \sigma_{r}^{*}$, and $\sigma_{\theta}^{*}$ in (51) are independent of $\nu$. See (7). 
addition, the complementary error function of complex argument $\psi\left(k^{1 / 2} \omega^{1 / 2}\right)$, in which $k$ is the complex parameter (38). According to (16) and (38),

$$
\left.\begin{array}{c}
\psi(k \omega)^{1 / 2}=\frac{2}{\pi^{1 / 2}} \int_{x}^{\infty} \exp \left(y^{2}-\eta^{2}-2 i y \eta\right) d \eta, \\
x=\left[\frac{\omega}{2 \gamma}\left(2^{1 / 2} p^{1 / 2}-p\right)\right]^{1 / 2}, \quad y=\left[\frac{\omega}{2 \gamma}\left(2^{1 / 2} p^{1 / 2}+p\right)\right]^{1 / 2},
\end{array}\right\}
$$

where $p$ and $q$ are given by (28).

While dense tabulations of $\psi(z)$, for real $z$, are available in [21], the existing tables for complex $z$ appear to be inadequate for our purposes. ${ }^{13}$ This fact, along with the general unwieldiness of (43), (45) and (47), complicates the numerical evaluation of the present results. The magnitude of $\gamma$ is bound to be exceedingly small compared to unity in applications which are of physical interest. ${ }^{14}$ It is natural, therefore, to seek

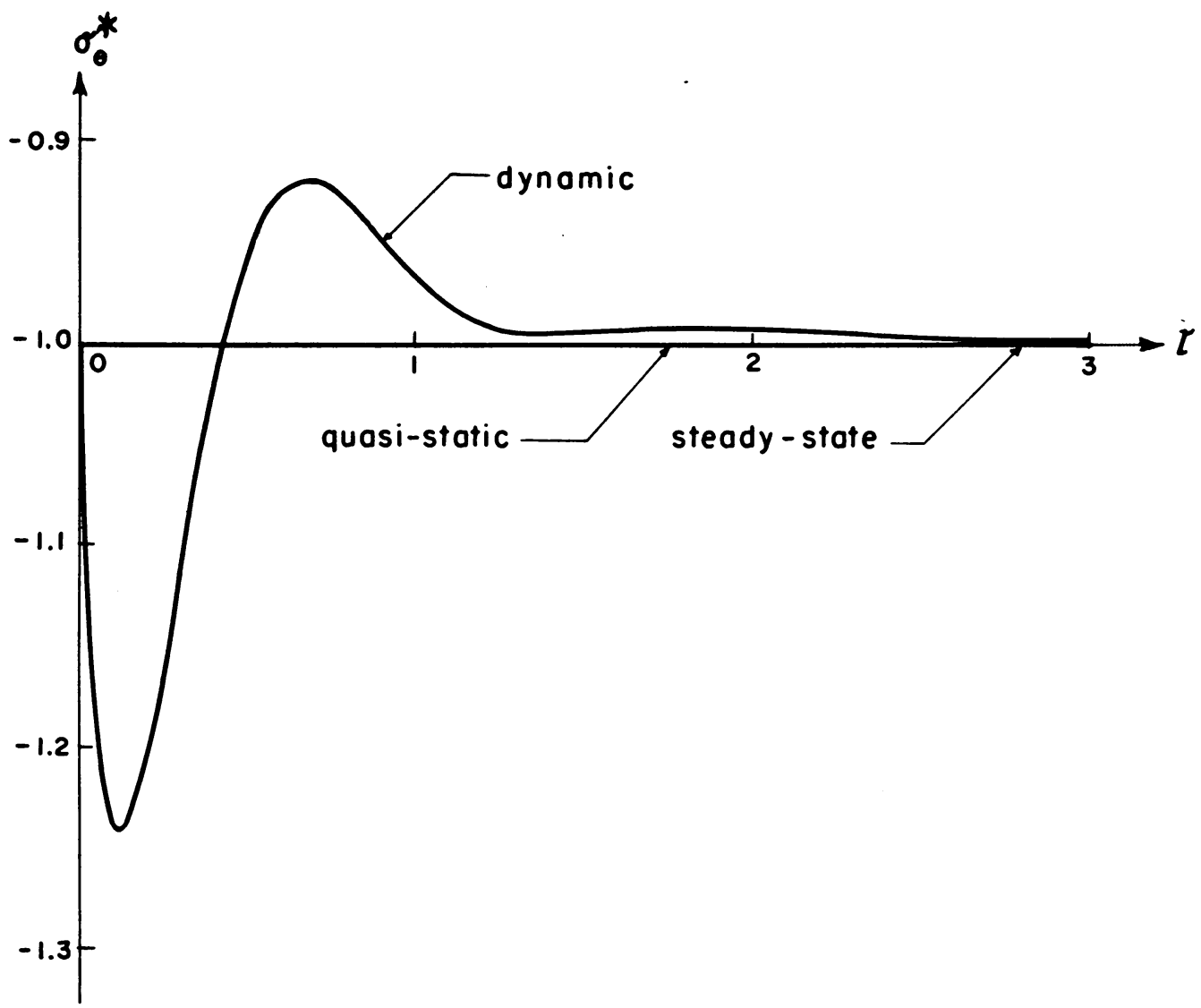

Fig. 1. Time-dependence of $\sigma_{\theta}^{*}$ at $\rho=1$ for $\gamma=1 / 5, \nu=1 / 4$.

${ }^{13}$ Approximate methods for the computation of the error function of complex argument are discussed by Rosser [22].

${ }^{14}$ If $a=1$ in. and the material is steel, $\gamma$ is of the order of $10^{-7}$. 


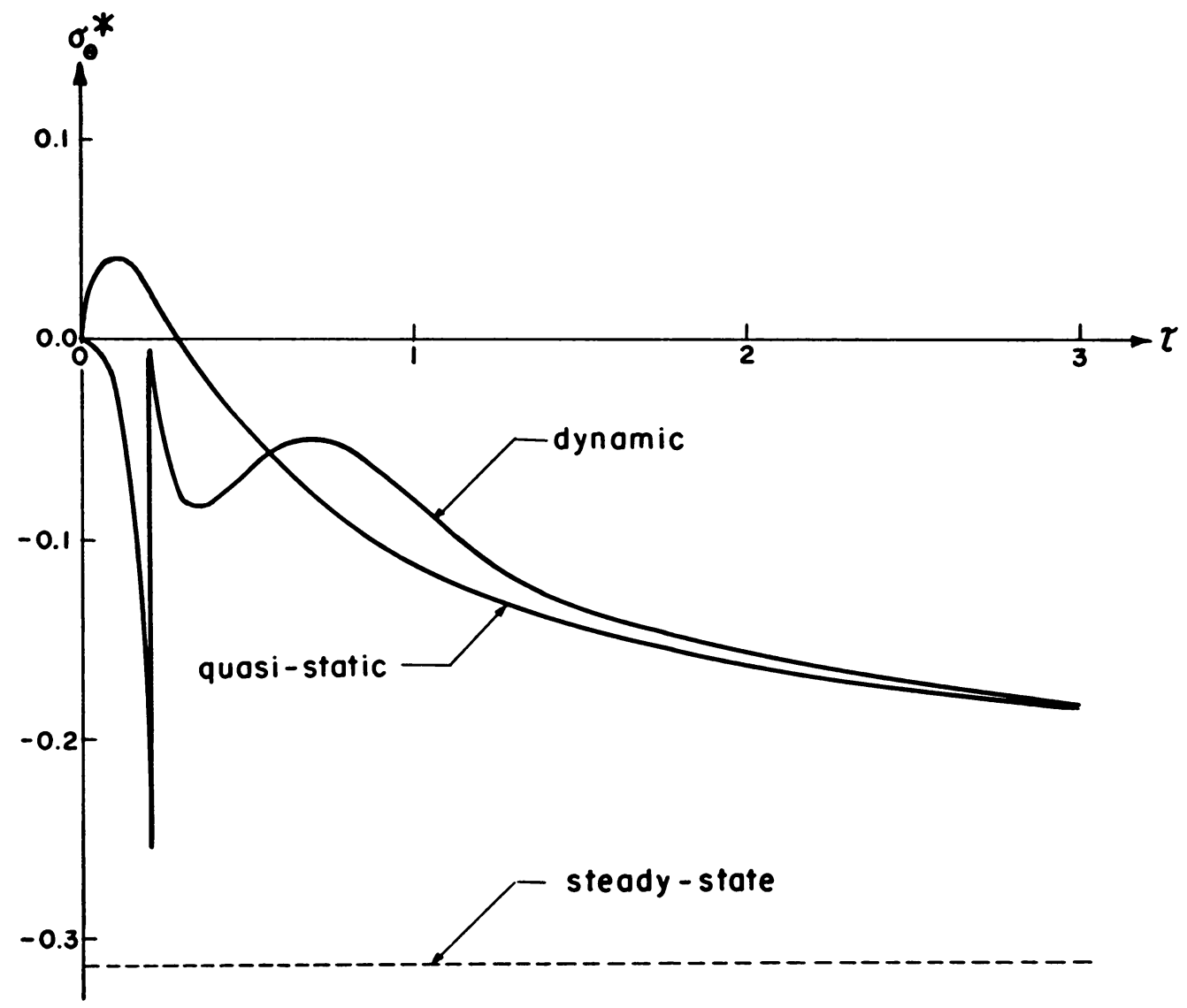

Fig. 2. Time-dependence of $\sigma_{\theta}^{*}$ at $\rho=2$ for $\gamma=1 / 5, \nu=1 / 4$.

asymptotic expansions for $u^{*}, \sigma_{r}^{*}$, and $\sigma_{\theta}^{*}$, in the neighborhood of $\gamma=0$, with the aid of the semi-convergent development (19). Unfortunately, the asymptotic expansions thus reached break down at $\rho=1$ and at $\tau=0$; for this reason, they fail to supply useful approximations to the solution in the most significant range of position and time.

Figures 1, 2, 3 show the time-dependence of $\sigma_{\theta}^{*}$ at $\rho=1,2,3$, respectively, for $\nu=1 / 4$ and $\gamma=1 / 5$. This unrealistically large value of the inertia parameter was chosen in order to bring out clearly the qualitative nature of the dynamic effects here involved. The underlying computations necessitated appropriate tabulations of the complex error function (54), which were carried out on a 650 I.B.M. electronic computer. The corresponding quasi-static curve $(\gamma=0)$, has been included on each of the diagrams under discussion.

In Fig. 1, the dynamic stress values are seen to undergo a pronounced oscillation, after which they approach the constant quasi-static value $\sigma_{\theta}^{*}(1, \tau)=-1$, which coincides with the steady-state boundary-value of $\sigma_{\theta}^{*}$. It is interesting to recall that the boundaryvalues of the transverse normal stress in Danilovskaya's problem [2], [5], do not exhibit any dynamic effects. In Figs. 2, 3, the dynamic curves display the jump discontinuity (49) at the wave-front and thereafter rapidly approach the corresponding quasi-static curves which, in turn, tend to the common steady-state asymptote. As the shock-wave 


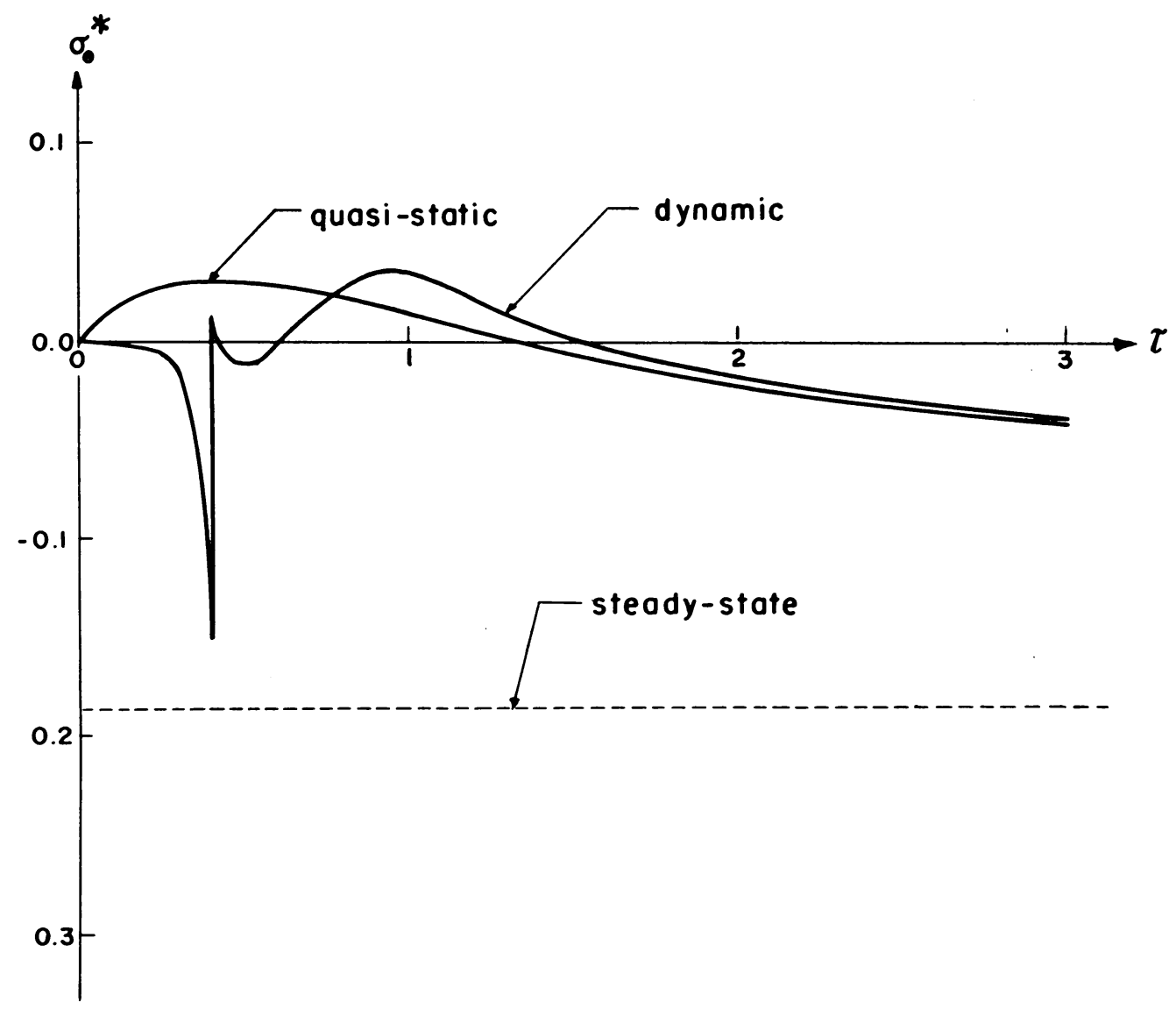

FIG. 3. Time dependence of $\sigma_{\theta}^{*}$ at $\rho=3$ for $\gamma=1 / 5, \nu=1 / 4$.

progresses, the difference between the values of $\sigma_{\theta}^{*}$ immediately behind and ahead of the wave-front decreases in accordance with (49); it should be kept in mind that this difference is independent of $\gamma$.

Figure 4 illustrates the quantitative influence of inertia upon the transient thermal stresses. Here, the dynamic peak-values of $\sigma_{\theta}^{*}$ on either side of the wave-front, and the quasi-static values at the wave-front, are plotted as a function of $\tau$. The dynamic curves are based on $\nu=1 / 4$ and $\gamma=7.3 \times 10^{-9}$. This choice of the parameters is descriptive of steel, if $a=1 \mathrm{ft}$. The current value of $\gamma$ is obtained from (8), (9), by use of the approximate values $\mu=5 \times 10^{10}$ poundals $/ \mathrm{ft}^{2}, \beta=484 \mathrm{lbs} / \mathrm{ft}^{3}, \kappa=1.28 \times 10^{-4} \mathrm{ft}^{2} / \mathrm{sec}$. In this instance $c=1.75 \times 10^{4} \mathrm{ft} / \mathrm{sec}$. Furthermore, for the preceding choice of $\alpha$ and $\kappa$, according to (2), $t=7.8 \times 10^{3} \tau$.

The curves appropriate to the dynamic $\sigma_{\theta}^{*}$ behind the wave-front $(\omega=0+)$ and to the quasi-static $\sigma_{\theta}^{*}$ at the wave-front $(\omega=0)$, are indistinguishable from the coordinate axes for the time-scale underlying the main diagram in Fig. 4. The inset diagram-drawn to a magnified time-scale - exhibits what might be called a boundary-layer effect with respect to time, which is characteristic of the behavior of $\sigma_{\theta}^{*}$ during the initial stage of the motion. Thus, the dynamic peak of $\sigma_{\theta}^{*}$ ahead of the wave-front is reduced to about one third of its initial limiting value of $-3 / 2$ during the first $4 \times 10^{-12}$ second of the 


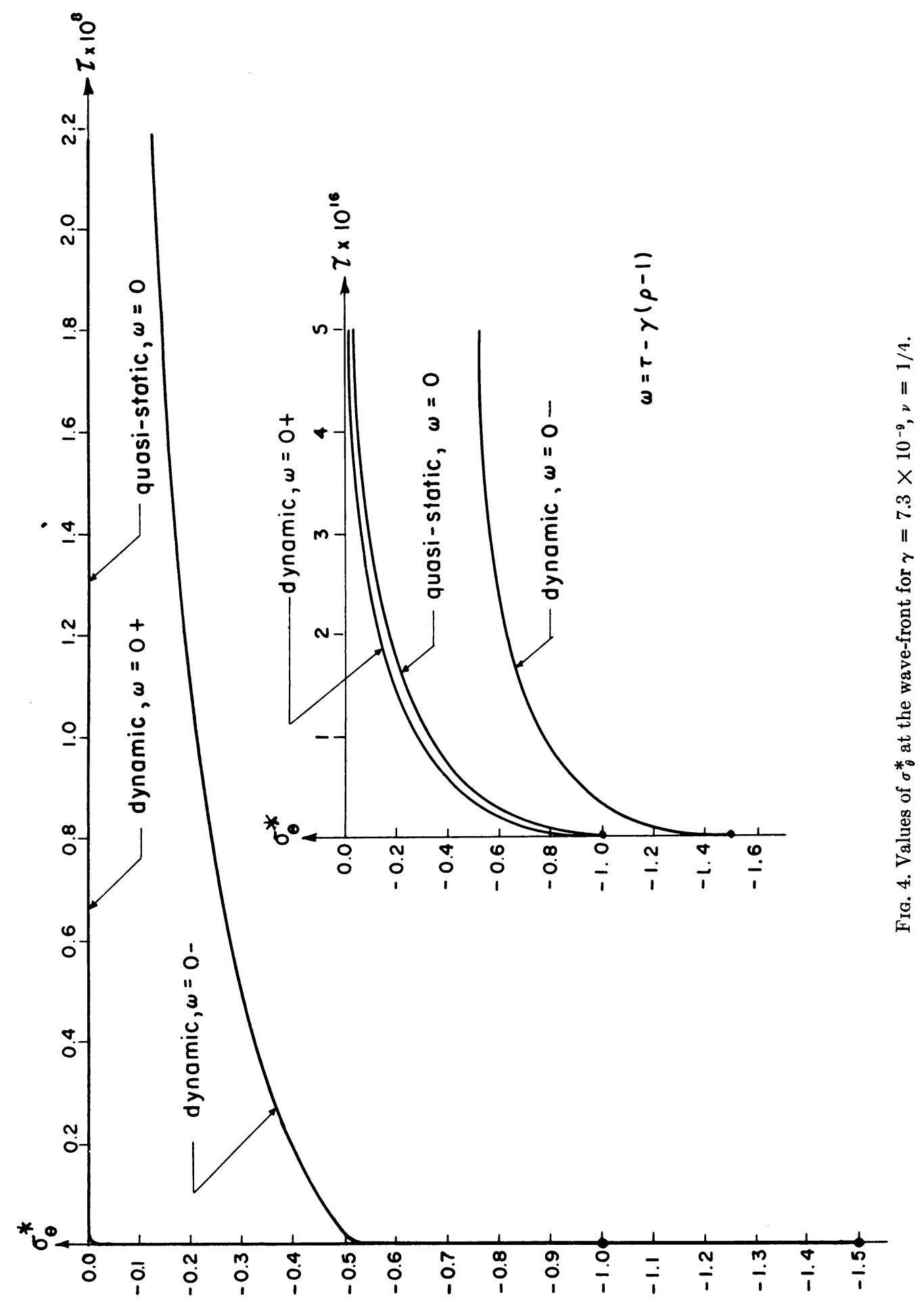


motion. In the same time-interval, the dynamic peak of $\sigma_{\theta}^{*}$ behind the wave-front as well as the quasi-static $\sigma_{\theta}^{*}$ at the front of the wave, decrease to less than five per cent of their joint initial value of -1 .

In conclusion we emphasize that the present results, which presuppose a stepfunction dependence upon time of the surface temperature, are bound to require severe modifications once the fiction of an instantaneous heating of the boundary is abandoned, even if the rate of heating, though finite, is extremely rapid by physically realistic standards. ${ }^{15}$

Acknowledgment. The authors are indebted to Mr. J. J. Kalker who conducted that portion of the numerical work which was carried out on the I.B.M. 650 electronic computer.

\section{REFERENCES}

1. J. M. C. Duhamel, Second mémoire sur les phénomènes thermoméchaniques, J. de l'Ecole Polytechnique $15,25,1$ (1837)

2. V. I. Danilovskaya, Thermal stresses in an elastic half-space due to a sudden heating of its boundary (in Russian), Prikl. Mat. Mekh. 14, 316 (1950)

3. T. Mura, Thermal strain and stresses in transient state, Proc. Second Japan Congr. Appl. Mech. 9 (1952)

4. V. I. Danilovskaya, On a dynamical problem of thermoelasticity (in Russian), Prikl. Mat. Mekh. 16, 3,341 (1952)

5. Eli Sternberg and J. G. Chakravorty, On inertia effects in a transient thermoelastic problem, Technical Report, Office of Naval Research, Contract Nonr 562(25), Brown University, May 1958. To appear in a future issue of the $\mathbf{J}$. Appl. Mech.

6. B. A. Boley, Thermally induced vibrations of beams, J. Aero. Sci. 23, 2, 179 (1956)

7. B. A. Boley and A. D. Barber, Dynamic response of beams and plates to rapid heating, J. Appl. Mech. 24, 3, 413 (1957)

8. W. Nowacki, A dynamical problem of thermoelasticity, Nadb. Arch. Mech. Stos. 9, 325 (1957)

9. J. Ignaczak, Thermal displacements in an elastic semi-space due to a sudden heating of the boundary plane, Nadb. Arch. Mech. Stos. 9, 4, 395 (1957)

10. Eli Sternberg, Transient thermal stresses in an infinite medium with a spherical cavity, Proc., Koninkl. Nederl. Akad. Wet., Ser. B, 60, 5, 396 (1957)

11. H. Jeffreys, On the cause of oscillatory movement in seismograms, Monthly Notices, Roy. Astron. Soc., Geo-phys. Suppl. 2, 8, 410 (1931)

12. H. S. Carslaw and J. C. Jaeger, Conduction of heat in solids, Clarendon Press, Oxford, 1947

13. Staff of the Bateman Manuscript Project, Higher transcendental functions, vol. 2, McGraw-Hill, New York, 1953

14. Staff of the Bateman Manuscript Project, Tables of integral transforms I, McGraw-Hill, New York, 1954

15. G. Grünberg, Über den in einer isotropen Kugel durch ungleichförmige Erwärmung erregten Spannungszustand, Z. Physik 35, 7, 548 (1926)

16. E. Melan, Mitteilung, Sitzungsbericht der math.-naturwiss. Klasse, Oesterr. Akad. Wiss., Oct. 13, 1955

17. E. Melan, Wärmespannungen bei der Abkühlung einer Kugel, Acta Physica Austriaca 10, 1-2, 81 (1956)

18. R. Trostel, Instationäre Wärmespannungen in einer Hohlkugel, Ingenieur-Archiv 24, 6, 373 (1956)

19. S. Timoshenko and J. N. Goodier, Theory of elasticity, 2nd. ed. McGraw-Hill, New York, 1951

20. E. L. McDowel and E. Sternberg, Axisymmetric thermal stresses in a spherical shell of arbitrary thickness, J. Appl. Mech. 24, 3, 376 (1957)

21. Tables of probability functions, vol. 1, New York W.P.A., 1941

22. J. B. Rosser, Theory and application of

22. J. B. Rosser, Theory and application of $\int_{0}^{z} e^{-x^{2}} d x$ and $\int_{0}^{z} e^{-p^{2} y^{2}} d y \int_{0}^{y} e^{-x^{2}} d x$, Mapleton House, New York, 1948.

${ }^{15}$ Compare the conclusions reached in [5]. 